\title{
Two-Stage Resistor-Load Logic for Digital Applications on Flexible Substrates
}

\author{
Hikmet Çeliker*†, Antony Sou ${ }^{\ddagger}$, Wim Dehaene* ${ }^{* \dagger}$ and Kris Myny* ${ }^{* \dagger}$ \\ ${ }^{*} \mathrm{KU}$ Leuven, Leuven, Belgium \\ †imec, Heverlee, Belgium \\ ${ }^{\ddagger}$ PragmatIC, Cambridge, United Kingdom \\ Hikmet.Celiker@imec.be
}

\begin{abstract}
A new standard cell topology, two-stage resistor-load logic, for Indium-Gallium-Zinc-Oxide (IGZO) thin-film transistor (TFT) technology is presented. The proposed inverter topology is compared with conventional one-stage resistor-load gates in terms of robustness (noise margin), speed performance and power consumption. It has been shown that the two-stage resistorload logic style is more robust against process variations and demonstrates higher speed performance. As a proof of concept, a 4-bit digital multiplier circuit on a flexible substrate is designed and fabricated with $1.66 \mathrm{~mm}^{2}$ area using $800 \mathrm{~nm}$ channel length n-type only TFTs. Measured power consumption of the chip is less than $12 \mathrm{~mW}$ with $1 \mathrm{MHz}$ of operating clock frequency. ${ }^{1}$
\end{abstract}

Keywords-TFT; digital design; logic topology; IGZO; inverter; flexible electronics

\section{INTRODUCTION}

Thin-film transistor (TFT) technologies have gained a key role in the last decades of semiconductor industry because of their unique properties. First, manufacturing in large areas became possible, paving the way for high resolution and large area displays. TFTs allow to use active matrix addressing techniques to switch individual pixels on the display [1]. Second, the process can be done on flexible substrates which brings a great potential for various form factors of displays and circuitry. One interesting research path is to connect everyday objects to the internet for IoT by means of flexible RFID tags [2]-[3]. Thanks to this flexible form of TFT circuits, on-body health patches for biomedical applications are also possible [4]-[5]. Third, TFTs using metal-oxide semiconductors such as Indium-Gallium-Zinc-Oxide (IGZO) have very low leakage current which enables them to be used in memory design for thin-film data processing circuits [6]-[7].

Developing process techniques to achieve sub-micron TFTs in parallel with new design methodologies pave the way for VLSI circuits on flexible substrates. In unipolar technologies, if dual-gate TFTs are not available, the conventional technique to create standard cells is to use one-stage resistor-load design as depicted in Figure 1a [8]. In this study, a two-stage resistorload topology as shown in Figure $1 \mathrm{~b}$ is proposed and compared with the one-stage design in terms of DC transfer characteristics and ring oscillator characteristics. In order to prove the

\footnotetext{
${ }^{1}$ This work has received funding from the European Research Council (ERC) under the European Union's Horizon 2020 research and innovation programme under grant agreement No 716426 (FLICs project).
}

concept, a 4-bit multiplier circuit on flexible substrate with two-stage resistor-load logic topology has been designed and fabricated in the $800 \mathrm{~nm}$ IGZO-based process technology of PragmatIC foundry. The average threshold voltage $\left(\mathrm{V}_{T}\right)$ of n-type-only transistors in this technology is $0.685 \mathrm{~V}$ with standard deviation of $57 \mathrm{mV}$. Unique aspects of this technology are the added resistor component that provides flexibility in circuit design, 4 routable metal layers to save circuit area, 800 $\mathrm{nm}$ channel lengths and rapid technology throughput time [9]. (a)

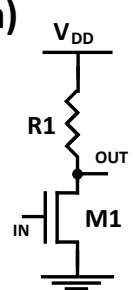

(b)

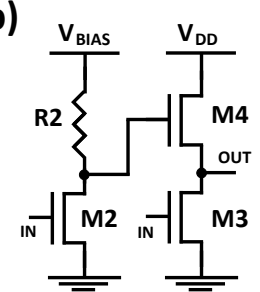

Fig. 1. Circuit schematics of 1-stage (a) and 2-stage (b) resistor-load inverters.

\section{DC TRANSFER CHARACTERISTICS}

The working principle of two-stage resistor-load logic is similar to Pseudo-CMOS logic [10]: The ratioed-logic at the first stage is a standard resistor-load inverter where the second stage comprises of a source follower and an additional input transistor. Supply voltage $\mathrm{V}_{D D}$ needs to be equal to or smaller than the supply voltage $\mathrm{V}_{B I A S}$ for a proper operation. Figure 2a compares voltage transfer curves of one-stage resistor-load and two-stage resistor-load inverters while Figure $2 b$ plots the extracted noise margin with respect to the supply voltage. It can be observed that the two-stage resistor-load inverter operates rail-to-rail and has a higher noise margin than the onestage resistor-load inverter independent from supply voltage. The output voltage of the one-stage inverter does not reach to zero volts due to the ratioed-logic property. This compromises the noise margin for all supply voltages [11]. The transistors have a W/L ratio of $50 \mu \mathrm{m} / 2 \mu \mathrm{m}$ and the resistance value of $\mathrm{R}_{1}$ and $\mathrm{R}_{2}$ is $200 \mathrm{k} \Omega(15 \mu \mathrm{m} / 3 \mu \mathrm{m})$. The area of an inverter cell with two-stage logic is $4050 \mu \mathrm{m}^{2}$ while it is $2250 \mu \mathrm{m}^{2}$ for one-state logic.

For each topology, three different designs were investigated with different resistance values for the load resistor $(100 \mathrm{k} \Omega$, $200 \mathrm{k} \Omega$ and $300 \mathrm{k} \Omega$ ). Figure $3 \mathrm{a}$ shows the voltage transfer 

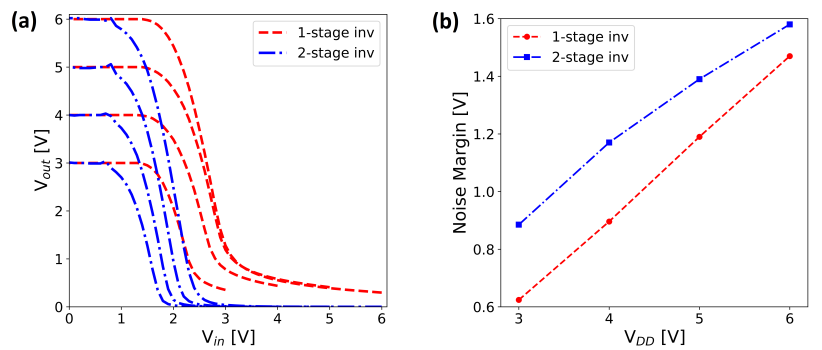

Fig. 2. Voltage transfer curves of inverters with different $\mathrm{V}_{D D}$ (a) and noise margin with respect to $\mathrm{V}_{D D}$ (b) $\left(\mathrm{V}_{D D}=\mathrm{V}_{B I A S}\right)$.

curves of two-stage resistor-load inverters with three different resistance values. Increasing the value of the load resistance makes the curve steeper and hence increases the noise margin. Figure $3 \mathrm{~b}$ plots the comparison between two logic styles in terms of average noise margin. Besides providing rail-to-rail output swing, another advantage of having the second stage is enabling tuning the voltage transfer curve by changing $\mathrm{V}_{B I A S}$ for the same $\mathrm{V}_{D D}$ as seen in Figure 4a. In this way, the noise margin of the inverter can be tuned to an optimum level after the fabrication of the circuit as shown in Figure $4 \mathrm{~b}$. The left $\mathrm{y}$-axis indicates the noise margin while the right $\mathrm{y}$-axis stands for the trip voltage where the input voltage is equal to the output voltage.
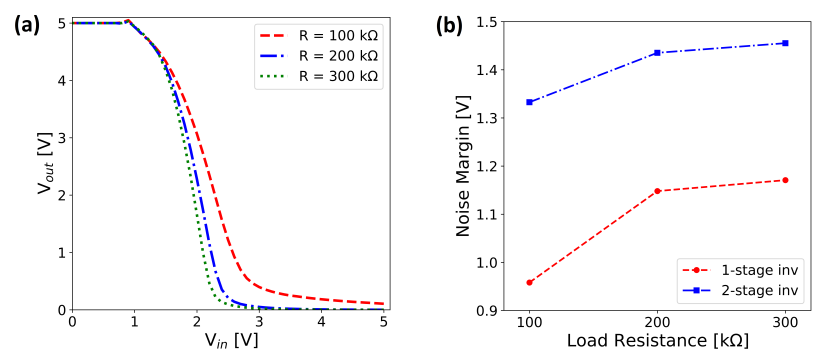

Fig. 3. Voltage transfer curves of 2-stage resistor-load inverter with resistance value of $100 \mathrm{k} \Omega, 200 \mathrm{k} \Omega$ and $300 \mathrm{k} \Omega$ (a) and noise margin with respect to load resistance value (b) $\left(\mathrm{V}_{D D}=\mathrm{V}_{B I A S}=5 \mathrm{~V}\right)$.
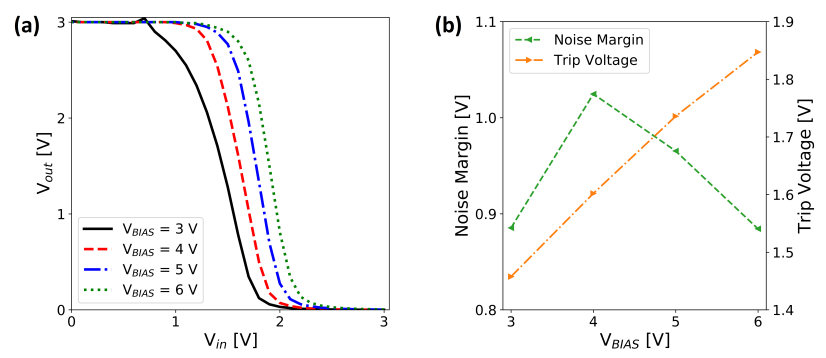

Fig. 4. Impact of $\mathrm{V}_{B I A S}$ on DC characteristics of 2-stage resistor-load inverter $\left(\mathrm{V}_{D D}=3 \mathrm{~V}\right)$.

According to Pelgrom's Law, threshold voltage mismatch between identically designed transistors is inversely proportional to the channel area of transistors [12]. In order to evaluate the robustness of smaller sized logic gates, two different inverter designs with sizes of TFTs W/L=50 $\mu \mathrm{m} / 2$ $\mu \mathrm{m}$ and $\mathrm{W} / \mathrm{L}=20 \mu \mathrm{m} / 0.8 \mu \mathrm{m}$ have been measured. The load resistor value for both inverters is identical $(200 \mathrm{k} \Omega)$ while the supply voltages are set to $5 \mathrm{~V}$. The average noise margin of the inverters with channel length of $2 \mu \mathrm{m}$ TFTs is measured as $1.435 \mathrm{~V}$ while it is $1.245 \mathrm{~V}$ for inverters with $\mathrm{L}=0.8$ $\mu \mathrm{m}$ TFTs. Considering that the threshold voltage variation of TFTs for the technology is $57 \mathrm{mV}$, logic gate design with $\mathrm{L}=$ $0.8 \mu \mathrm{m}$ is promising for complex circuit integration and faster operating circuits.

\section{Ring Oscillator Characteristics}

In order to evaluate the speed performance and power consumption of the standard cell topology, ring oscillator circuits with 19 cascaded inverters are designed and measured. Figure 5a shows the average propagation delay and power consumption per inverter for different supply voltage combinations based on designs with $\mathrm{L}=2 \mu \mathrm{m}$. As the red curve demonstrates, one-stage resistor-load inverters have a tradeoff between speed and power consumption and the delay is reduced with the increasing supply voltage. Two-stage resistorload inverters have a slightly different behavior due to the second supply rail. In Figure 5a, while going from point $A$ to point $\mathrm{B}$ (solid line in the blue curve), $\mathrm{V}_{D D}$ and $\mathrm{V}_{B I A S}$ are equally increased from $2 \mathrm{~V}$ to $5 \mathrm{~V}$. From point $\mathrm{A}$ to point $\mathrm{C}$ (dotted line), $\mathrm{V}_{D D}$ is kept at $2 \mathrm{~V}$ and $\mathrm{V}_{B I A S}$ is increased to 5 $\mathrm{V}$. Lastly, from point $\mathrm{B}$ to point $\mathrm{C}$ (dash-dotted line), $\mathrm{V}_{B I A S}$ is kept at $5 \mathrm{~V}$ and $\mathrm{V}_{D D}$ is reduced from $5 \mathrm{~V}$ to $2 \mathrm{~V}$. It can be observed that two-stage resistor-load inverter can operate at higher speeds with different $\mathrm{V}_{D D}$ and $\mathrm{V}_{B I A S}$ combinations.
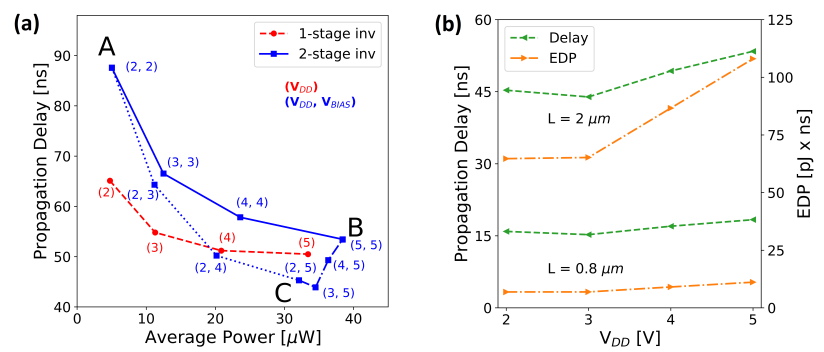

Fig. 5. Propagation delay for different supply voltages (a) and delay \& EDP vs $\mathrm{V}_{D D}$ for two-stage resistor-load inverter (b).

Designing transistors as small as the processing technology allows is necessary in order to have the maximum operating speed, the lowest power consumption and the smallest circuit area for a digital circuit. Figure 5b shows the impact of transistor size on propagation delay (y-axis on left) and energy $\mathrm{x}$ delay product (y-axis on right) for different $\mathrm{V}_{D D}$ values while $\mathrm{V}_{B I A S}$ is kept at $5 \mathrm{~V}$. For both designs $(\mathrm{L}=2 \mu \mathrm{m}$ and $\mathrm{L}=0.8 \mu \mathrm{m})$, an average of four different samples around the wafer showed that the minimum propagation delay is achieved when $\mathrm{V}_{D D}$ is equal to $3 \mathrm{~V}$. Channel length scaling from $2 \mu \mathrm{m}$ to $0.8 \mu \mathrm{m}$ with the same aspect ratio reduces the propagation delay from 43.9 to $15.2 \mathrm{~ns}$ and EDP from 65.2 to $6.9 \mathrm{pJ} \mathrm{x}$ $n s$. 


\section{4-Bit Multiplier Design And Measurement RESULTS}

To further evaluate the two-stage resistor-load topology and demonstrate the proof of concept, a 4-bit digital multiplier was designed and fabricated. After generating a standard cell library which contains 8 cells (from simple NOR2 to more complex Or-And-Inv-2-1), a typical digital design flow procedure for TFTs is followed from RTL to GDS level similar to CMOS physical implementation flows. Schematics of standard cells are based on the inverter in Figure 1b with $\mathrm{R}$ equal to $200 \mathrm{k} \Omega, \mathrm{W}$ is $20 \mu \mathrm{m}$ and $\mathrm{L}$ is $800 \mathrm{~nm}$ for TFTs.

The scan-chain method is used for testability purposes. Figure 6a details the block diagram of the designed 4-bit multiplier with scan chain. The testing method of the circuit is as follows: 16 negative edge triggered flip-flops are filled in through the 'Scan In' input pin with the values of two 8bit input sets. Each input set consists of two 4-bit numbers to be multiplied with each other. After all inputs are ready, the 'Scan Enable' pin -which is connected to select pins of multiplexers- is brought to logic 0 and the multiplier is in its operating mode. While in this state, two fast clock pulses are given so that the output flip-flops are filled in with the result of the second multiplication. Then 'Scan Enable' pin is returned to logic 1 and the 8-bit result is read out through the 'Scan Out' pin starting from the most significant bit with slow clock pulses. (a)

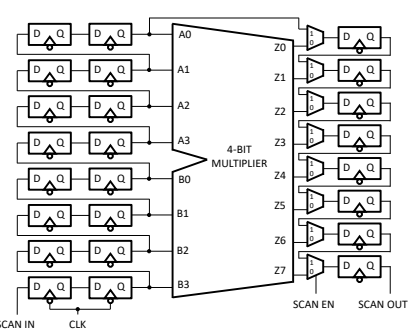

(b)

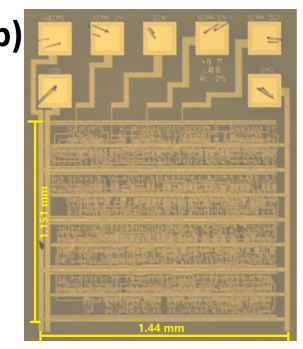

Fig. 6. Block diagram of 4-bit multiplier with scan chain (a) and microscope image (b).

Figure $6 \mathrm{~b}$ shows the microscope image of the flexible chip whose area is $1.66 \mathrm{~mm}^{2}$ with width of $1440 \mu \mathrm{m}$ and length of $1151.5 \mu \mathrm{m}$ excluding the test pads. The total number of TFTs in the chip is 1351 while the number of resistor components is 257 . Standard cells constitute 75 percent of this area while the rest is used for routing of signals and power. As it is a 4-metal thin-film technology, wiring is performed on top of the standard cell in the final chip layout. The supplies of the standard cells are aligned back to back and do not use routing channels in the lower metal layers, as can be seen in Figure $6 b$.

The critical path of the 4-bit multiplier is found via SPICE simulation based on the synthesized gate-level circuit netlist. Table I shows the input sequence for the critical path. The critical path which can be measured on the chip is equal to $t_{C l k-t o-Q}+t_{d-\text { multiplication }}+t_{d-\text { multiplexer }}+t_{\text {setup-FF }}$.

Figure $7 \mathrm{a}$ is the Shmoo plot for the multiplier circuit for different clock frequencies and supply voltages. Figure 8
TABLE I

INPUT SEQUENCE FOR THE CRITICAL PATH

\begin{tabular}{|c|c|c|c|c|}
\cline { 2 - 5 } \multicolumn{1}{c|}{} & \multicolumn{2}{c|}{ First Multiplication } & \multicolumn{2}{c|}{ Second Multiplication } \\
\cline { 2 - 5 } \multicolumn{1}{c|}{} & Decimal & Binary & Decimal & Binary \\
\hline A & 5 & 0101 & 6 & 0110 \\
\hline B & 10 & 1010 & 11 & 1011 \\
\hline Out & 50 & 00110010 & 66 & 01000010 \\
\hline
\end{tabular}

shows the scope data for measurement at $1 \mathrm{MHz}$ clock speed when $\mathrm{V}_{D D}$ is $3 \mathrm{~V}$ and $\mathrm{V}_{B I A S}$ is $6 \mathrm{~V}$. To make sure that the input data is loaded to the flip-flops and the output data is read-out properly, the slow clock frequency is kept at $100 \mathrm{~Hz}$ for all cases. The average power consumption values of the different samples for various supply voltage combinations are shown in Figure $7 \mathrm{~b}$. It should be noted that the contribution of dynamic power to the total power is negligible due to the dominant static current of this unipolar topology.
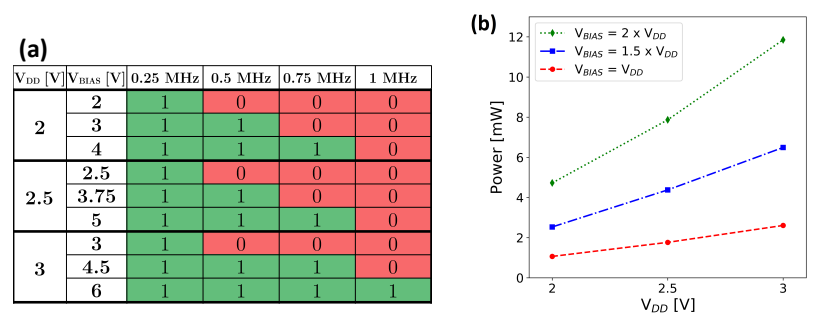

Fig. 7. Shmoo plot for 4-bit multiplier (1: pass, 0: fail) (a) and power consumption of 4-bit multiplier for different supply voltage values (b).

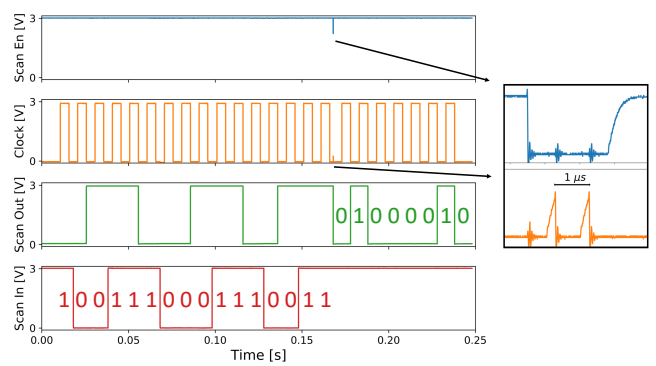

Fig. 8. Scope data from 4-bit multiplier.

\section{CONCLUSION}

A new logic topology, two-stage resistor-load, for digital design applications with IGZO TFT technology is proposed. This logic topology is compared with one-stage resistor-load logic with respect to the DC transfer characteristics and the ring oscillator behavior of inverters. Even though two-stage resistor-load topology requires more area and an extra pin for the power supply, it shows a better performance than onestage resistor-load logic. Rail-to-rail operation of two-stage resistor-load topology results higher noise margin and tuning the second supply voltage enables to compensate the impacts of global variability on circuit robustness. A standard cell library based on the proposed two-stage resistor-load logic topology is created and a 4-bit multiplier on flexible substrate is fabricated and tested showing an outstanding performance also in a larger circuit. 


\section{REFERENCES}

[1] J.-H. Lee, J.-H. Kim, and M.-K. Han, "A new a-si: H tft pixel circuit compensating the threshold voltage shift of a-si: $\mathrm{H}$ tft and oled for active matrix oled," IEEE electron device letters, vol. 26, no. 12, pp. 897-899, 2005.

[2] K. Myny, "The development of flexible integrated circuits based on thin-film transistors," Nature Electronics, vol. 1, no. 1, pp. 30-39, 2018.

[3] M.-H. Hung, C.-H. Chen, Y.-C. Lai, K.-W. Tung, W.T. Lin, H.-H. Wang, F.-J. Chan, C.-C. Cheng, C.-T. Chuang, Y.-S. Huang et al., "Ultra low voltage 1-v rfid tag implement in a-igzo tft technology on plastic," in 2017 IEEE International Conference on RFID (RFID). IEEE, 2017, pp. 193-197.

[4] M. Zulqarnain, S. Stanzione, J.-L. P. Van Der Steen, G. H. Gelinck, K. Myny, S. Abdinia, and E. Cantatore, "A $52 \mu \mathrm{w}$ heart-rate measurement interface fabricated on a flexible foil with a-igzo tfts," in ESSCIRC 2018IEEE 44th European Solid State Circuits Conference (ESSCIRC). IEEE, 2018, pp. 222-225.

[5] M. Sugiyama, T. Uemura, M. Kondo, M. Akiyama, N. Namba, S. Yoshimoto, Y. Noda, T. Araki, and T. Sekitani, "An ultraflexible organic differential amplifier for recording electrocardiograms," Nature Electronics, vol. 2, no. 8, pp. 351-360, 2019.

[6] F. De Roose, K. Myny, M. Ameys, J.-L. P. van der Steen, J. Maas, J. de Riet, J. Genoe, and W. Dehaene, "A thinfilm, a-igzo, 128b sram and lprom matrix with integrated periphery on flexible foil," IEEE Journal of Solid-State Circuits, vol. 52, no. 11, pp. 3095-3103, 2017.

[7] Y. Kurokawa, Y. Okamoto, T. Nakagawa, T. Aoki, M. Ikeda, M. Kozuma, T. Osada, T. Ikeda, N. Yamade, Y. Okazaki et al., "Applications of crystalline indiumgallium-zinc-oxide technology to lsi: Memory, processor, image sensor, and field programmable gate array," in Fifth Asia Symposium on Quality Electronic Design (ASQED 2013). IEEE, 2013, pp. 66-71.

[8] E. Ozer, J. Kufel, J. Myers, J. Biggs, G. Brown, A. Rana, A. Sou, C. Ramsdale, and S. White, "A hardwired machine learning processing engine fabricated with submicron metal-oxide thin-film transistors on a flexible substrate," Nature Electronics, vol. 3, no. 7, pp. 419-425, 2020.

[9] "Flexlogic $\AA \quad$ fab." [Online]. Available: https://www.pragmaticsemi.com/create-more/devices

[10] T.-C. Huang, K. Fukuda, C.-M. Lo, Y.-H. Yeh, T. Sekitani, T. Someya, and K.-T. Cheng, "Pseudo-cmos: A design style for low-cost and robust flexible electronics," IEEE Transactions on Electron Devices, vol. 58, no. 1, pp. 141-150, 2010.

[11] K. Abbas, "Ratioed logic," in Handbook of Digital CMOS Technology, Circuits, and Systems. Springer, 2020, pp. 81-109.

[12] M. J. Pelgrom, A. C. Duinmaijer, and A. P. Welbers, "Matching properties of mos transistors," IEEE Journal of solid-state circuits, vol. 24, no. 5, pp. 1433-1439, 1989. 